\title{
q-ALGEBRA REPRESENTATIONS OF THE EUCLIDEAN, PSEUDO-EUCLIDEAN AND OSCILLATOR ALGEBRAS, AND THEIR TENSOR PRODUCTS
}

\author{
E.G. KALNINS $\dagger$ AND WILLARD MILLER, JR. $\ddagger$
}

\begin{abstract}
This is a discussion of various $q$-analogs of the euclidean and pseudo-euclidean groups and Lie algebras in 2-space, and of the oscillator algebra. We will work out some tensor products of irreducible representations of these algebras, compute the Clebsch-Gordan coefficients and derive the associated special function identities that arise. Cases where $0<$ $q<1,|q|=1$ and $q^{n}=1$ will be considered.
\end{abstract}

PACS: $02.20 .+\mathrm{b}, 03.65 . \mathrm{Fd}$

1. Introduction. Our paper is motivated by the factorization method of DarbouxSchrödinger-Infeld-Hull. [1,2]. This method provides a means of computing the eigenvalues and eigenfunctions for families of Schrödinger operators in quantum mechanics. For 30 years now it has been known that the factorization method is intimately related to the representation theory of the following Lie algebras: the euclidean Lie algebra in the plane $m(2)$, the harmonic oscillator algebra, the special unitary algebra $s u(2)$, the euclidean Lie algebra in 3 -space $m(3)$, and the special orthogonal Lie algebra $s o(4),[3,4]$. (In this paper we will restrict consideration to the first two of these algebras and their $q$-analogs.) The connection with representation theory provides a means of computing addition formulas and Clebsch-Gordan type expansions for the eigenfunctions that do not follow from the factorization method by itself. Although these results apply only to the Lie algebras of first order differential operators obtainable from the factorization method, this method can also be used to obtain factorizations of difference and $q$-difference equations, e.g. [5-8]. In just the same way the representation theory of $q$-algebras and quantum groups can be used to obtain properties of these $q$-factorizations.

In $\S 2$ we will briefly review relationships between the representation theory of $m(2)$ and the oscillator algebra, and properties of Bessel and Laguerre functions. The remainder of the paper will be devoted to preliminary results on various $q$-analogs of these algebras and

1991 Mathematics Subject Classification. 33D55, 33D45, 17B37, 81R50.

Key words and phrases. basic hypergeometric functions, $q$-algebras, quantum groups, difference operators. $\dagger$ Department of Mathematics and Statistics, University of Waikato, Hamilton, New Zealand. e-mail: MATH0236@waikato.ac.nz

¥School of Mathematics and Institute for Mathematics and its Applications, University of Minnesota, Minneapolis, Minnesota 55455. e-mail: miller@ima.umn.edu, Work supported in part by the National Science Foundation under grant DMS 94-00533 
their representation theories. In each case the raising and lowering operators $E_{ \pm}$can be used to determine factorizations associated with the representation. The notation used for $q$-series and $q$-integrals in this paper follows that of Gasper and Rahman [9].

2. $m(2)$ and oscillator algebra representations. The three dimensional Lie algebra $m(2)$ is determined by its generators $H, E_{+}, E_{-}$which obey the commutation relations

$$
\left[H, E_{+}\right]=E_{+}, \quad\left[H, E_{-}\right]=-E_{-}, \quad\left[E_{+}, E_{-}\right]=0 .
$$

We consider irreducible representations $(\omega)$ of $m(2)$, characterized by the positive number $\omega$. The spectrum of $H$ corresponding to $(\omega)$ is the set $Z=\{m: m$ an integer $\}$ and the complex representation space has basis vectors $f_{m}, m \in Z$, such that

$$
E_{ \pm} f_{m}=\omega f_{m \pm 1}, H f_{m}=m f_{m}, E_{+} E_{-} f_{m}=\omega^{2} f_{m}
$$

where $C \equiv E_{+} E_{-}$is an invariant operator. A simple realization of $(\omega)$ is given by the operators

$$
H=z \frac{d}{d z}, E_{+}=\omega z, E_{-}=\frac{\omega}{z}
$$

acting on the space of all linear combinations of the functions $z^{n}, z$ a complex variable, $n \in Z$, with basis vectors $f_{m}(z)=z^{m}$.

We can introduce an inner product such that $<f_{n}, f_{n^{\prime}}>=\delta_{n n^{\prime}}, n, n^{\prime} \in Z$. On the dense subspace $\mathcal{K}$ of all finite linear combinations of the basis vectors we have

$$
<E_{+} f, f^{\prime}>=<f, E_{-} f^{\prime}>, \quad<H f, f^{\prime}>=<f, H f^{\prime}>
$$

for all $f, f^{\prime} \in \mathcal{K}$, so $H=H^{*}$ and $E_{+}^{*}=E_{-}$. In terms of the operators (2.3) we can obtain a realization of $(\omega)$ and its Hilbert space structure by setting $z=e^{i \theta}$ :

$$
\begin{gathered}
H=-i \frac{d}{d \theta}, E_{+}=\omega e^{i \theta}, E_{-}=\omega e^{-i \theta} \\
f_{n}(z)=e^{i n \theta},<f, f^{\prime}>=\frac{1}{2 \pi} \int_{0}^{2 \pi} f\left(e^{i \theta}\right) \overline{f^{\prime}\left(e^{i \theta}\right)} d \theta .
\end{gathered}
$$

Matrix elements $T_{m^{\prime} m}$ of the complex motion group in the representation $(\omega)$ are typically defined by the expansions

$$
e^{\beta E_{+}} e^{\alpha E_{-}} e^{\tau H} f_{m}=\sum_{m^{\prime}=-\infty}^{\infty} T_{m^{\prime} m}(\alpha, \beta, \tau) f_{m^{\prime}}
$$

[4,10]. Explicitly,

$$
T_{m^{\prime} m}(\alpha, \beta, \tau)=\frac{e^{\tau m}(\alpha \omega)^{m-m^{\prime}}}{\Gamma\left(m-m^{\prime}+1\right)}{ }_{0} F_{1}\left(m-m^{\prime}+1 ; \omega^{2} \alpha \beta\right)=e^{\left[\tau m+i(\pi / 2+\theta)\left(m-m^{\prime}\right)\right]} J_{m-m^{\prime}}(\omega r)
$$


where $\alpha=i r e^{i \theta} / 2, \beta=i r e^{-i \theta} / 2$ and $J_{n}(r)$ is a Bessel function. The group multiplication property of the operators on the left-hand side of (2.6) leads to addition theorems for the matrix elements. For convenience in the computations to follow we shall limit ourselves to the case where $\tau=0$.

We define the tensor product $\left(\omega_{1}\right) \otimes\left(\omega_{2}\right)$ of two irreducible representations $\left(\omega_{1}\right)$ and $\left(\omega_{2}\right)$ by the operators $F_{+}, F_{-}, L$ :

$$
F_{ \pm}=E_{ \pm} \otimes I+I \otimes E_{ \pm}, \quad L=H \otimes I+I \otimes H
$$

Here, $I$ is the identity operator on the corresponding representation space. The operators $F_{+}, F_{-}, L$ again obey the commutation relations $(2.1)$ and the representation is unitary. It is straightforward to decompose this representation into a direct integral of irreducible representations, [10]:

$$
\left(\omega_{1}\right) \oslash\left(\omega_{2}\right) \cong \oplus \frac{1}{2 \pi} \int_{0}^{2 \pi}\left(\omega_{\mu}\right) d \mu
$$

where $\omega_{\mu}^{2}=\omega_{1}^{2}+\omega_{2}^{2}+2 \omega_{1} \omega_{2} \cos \mu$. From the proof of (2.9) we see that the matrix elements of the operator $e^{\beta F_{+}} e^{\alpha F_{-}}$with respect to the tensor product basis $f_{n}^{\omega_{1}} \otimes f_{n^{\prime}}^{\omega_{2}}$ and with respect to the reduced basis $f_{m}^{\omega_{\mu}}$ are related by the formula

$$
J_{n^{\prime}}\left(r^{\prime}\right) J_{n}\left(r^{\prime \prime}\right)=\frac{1}{2 \pi} \int_{0}^{2 \pi} e^{i\left(n \phi^{\prime \prime}-n^{\prime} \phi+n \phi\right)} J_{n+n^{\prime}}(r) d \phi^{\prime \prime}
$$

where $r e^{i \phi}=r^{\prime}+r^{\prime \prime} e^{i \phi^{\prime \prime}}$.

The oscillator algebra is the 4-dimensional Lie algebra generated by the elements $H$, $E_{+}, E_{-}, \mathcal{E}$ that obey the commutation relations

$$
\left[H, E_{+}\right]=E_{+},\left[H, E_{-}\right]=-E_{-},\left[E_{+}, E_{-}\right]=-\mathcal{E},\left[\mathcal{E}, E_{ \pm}\right]=[\mathcal{E}, H]=0 .
$$

It admits a class of algebraically irreducible representations $\uparrow_{\lambda, \ell^{2}}$ where $\ell, \lambda$ are real numbers and $\ell>0$. These are defined on a vector space with orthogonal basis $\left\{f_{m}: n=\right.$ $0,1, \cdots\}$ where

$$
E_{+} f_{m}=\ell f_{m+1}, E_{-} f_{m}=\ell m f_{m-1}, H f_{m}=(-\lambda+m) f_{m} \mathcal{E} f_{m}=\ell^{2} f_{m}
$$

Here, $C f_{m}=\lambda \ell^{2} f_{m}$ where $C=E_{+} E_{-}-\mathcal{E} H$ is an element in the center of the Lie algebra.

The matrix elements $T_{m^{\prime} m}(a, b)$ of the operator $e^{a E_{+}} e^{b E_{-}}$are defined by

$$
e^{a E_{+}} e^{b E_{-}} f_{m}=\sum_{m^{\prime}=0}^{\infty} T_{m^{\prime} m}(a, b) f_{m^{\prime}}
$$

$[4,10,11]$, where,

$$
T_{m^{\prime} m}(a, b)=(\ell b)^{m-m^{\prime}} L_{m^{\prime}}^{\left(m-m^{\prime}\right)}\left(-\ell^{2} a b\right)
$$


and $L_{n}^{(m)}(x)$ is an associated Laguerre polynomial. The tensor product $\uparrow_{\lambda_{1}, \ell_{1}^{2}} \otimes \uparrow_{\lambda_{2}, \ell_{2}^{2}}$ is defined by the coproduct

$$
\begin{aligned}
F_{ \pm} & =E_{ \pm} \otimes I+I \otimes E_{ \pm}, \quad L=H \otimes I+I \otimes H \\
\mathcal{F} & =\mathcal{E} \otimes I+I \otimes \mathcal{E}, \quad C=F_{+} F_{-}-\mathcal{F} L
\end{aligned}
$$

The operators $F_{+}, F_{-}, L, \mathcal{F}$ again obey the commutation relations (2.11) and the representation is unitary. (Here, $C$ is the invariant operator.) It is straightforward to decompose this representation into a direct sumof irreducible representations, $[4,11]$ :

$$
\uparrow_{\lambda_{1}, \ell_{1}^{2}} \otimes \uparrow_{\lambda_{2}, \ell_{2}^{2}} \cong \sum_{s=0}^{\infty} \oplus \uparrow_{\lambda_{1}+\lambda_{2}-s, \ell_{1}^{2}+\ell_{2}^{2}}
$$

The Clebsch-Gordan coefficients are expressible in terms of hypergeometric functions ${ }_{2} F_{1}$ and the identity relating the matrix elements of the operator $e^{\beta F_{+}} e^{a F_{-}}$with respect to the tensor product basis and with respect to the reduced basis takes the symbolic form ${ }_{1} F_{1} \times{ }_{1} F_{1} \sim \sum{ }_{2} F_{1} \times{ }_{1} F_{1} \times{ }_{2} F_{1}$.

Since the representation $(\omega)$ of $m(2)$ defines a representation of the oscillator algebra for which $\mathcal{E}=0$ we can also consider the tensor product $(\omega) \otimes \uparrow_{\lambda, \ell^{2}}$, defined by expressions (2.13). The result is [4]

$$
(\omega) \otimes \uparrow_{\lambda, \ell^{2}} \cong \sum_{n=-\infty}^{\infty} \oplus \uparrow_{\lambda+n, \ell^{2}},
$$

and the identity relating the matrix elements of the operator $e^{\beta F_{+}} e^{a F_{-}}$with respect to the tensor product basis and with respect to the reduced basis takes the form

$$
e^{\rho^{2}} r^{s-w} J_{w-s}(2 \rho r) L_{q}^{(k-q)}\left(r^{2}\right)=\sum_{n} \frac{i^{s-w} k !}{(n+k-s) !} L_{q}^{(n-w)}(\rho) L_{k}^{(n-s)}(\rho) L_{n+q-w}^{(k-q+w-s)}\left(r^{2}\right) \rho^{2 n-w-s} .
$$

3. $q$-analogs of $m(2)$ and the oscillator algebra. In addition to the standard definition of the tensor product of two irreducible representations $\left(\omega_{1}\right)$ and $\left(\omega_{2}\right)$ of $m(2)$ there is a definition given by the nontrivial Hopf algebra structure in which the coproduct is [12-15]

$$
F_{+}=E_{+} \otimes q^{-\frac{1}{2} H}+q^{\frac{1}{2} H} \otimes E_{+}, F_{-}=E_{-} \otimes q^{-\frac{1}{2} H}+q^{\frac{1}{2} H} \otimes E_{-} L=H \otimes I+I \otimes H
$$

where $0<q<1$. The operators $F_{ \pm}, L$ satisfy the commutation relations (2.1). The standard definition is obtained by letting $q \rightarrow 1$ in (3.1). With the $q$-analogs of the exponential function

$$
e_{q}(x)=\sum_{k=0}^{\infty} \frac{x^{k}}{(q ; q)_{k}}=\frac{1}{(x ; q)_{\infty}},|x|<1 ; \quad E_{q}(x)=\sum_{k=0}^{\infty} \frac{q^{k(k-1) / 2} x^{k}}{(q ; q)_{k}}=(-x ; q)_{\infty},
$$

we define the following $q$-analogs of matrix elements of $(\omega),[13,15,16]$ :

$$
e_{q}\left(\beta E_{+}\right) E_{q}\left(\alpha E_{-}\right) f_{n}=\sum_{n^{\prime}=-\infty}^{\infty} T_{n^{\prime} n}^{(e, E)}(\alpha, \beta) f_{n^{\prime}},|\omega \beta|<1 .
$$


Explicitly,

$$
\begin{aligned}
& T_{n^{\prime} n}^{(e, E)}(\alpha, \beta)=\frac{\left(q^{n-n^{\prime}+1} ; q\right)_{\infty}(\alpha \omega)^{n-n^{\prime}}}{(q ; q)_{\infty}} q^{\left(n-n^{\prime}\right)\left(n-n^{\prime}-1\right) / 2}{ }_{1} \phi_{1}\left(\begin{array}{c}
0 \\
q^{n-n^{\prime}+1} ; q,-\alpha \beta \omega^{2} q^{n-n^{\prime}}
\end{array}\right) \\
& =\frac{\left(q^{n^{\prime}-n+1} ; q\right)_{\infty}(\beta \omega)^{n^{\prime}-n}}{(q ; q)_{\infty}}{ }_{\infty} \phi_{1}\left(\begin{array}{c}
0 \\
q^{n^{\prime}-n+1}
\end{array} ; q,-\alpha \beta \omega^{2}\right) .
\end{aligned}
$$

If $\alpha \beta \neq 0$ we can express these results in terms of the Hahn-Exton $q$-Bessel function $[9,13]$

$$
J_{\nu}(z ; q)=\frac{\left(q^{\nu+1} ; q\right)_{\infty}}{(q ; q)_{\infty}} z^{\nu}{ }_{1} \phi_{1}\left(\begin{array}{c}
0 \\
q^{\nu+1} ; q, q z^{2}
\end{array}\right)
$$

Indeed, setting $\alpha=i r e^{i \psi}, \beta=i r e^{-i \psi}$, we see that in terms of the new complex coordinates $\left[r, e^{i \psi}\right]$ we have

$$
T_{n^{\prime} n}^{(e, E)}\left[r, e^{i \psi}\right]=e^{i\left(\frac{\pi}{2}-\psi\right)\left(n^{\prime}-n\right)} q^{\left(n^{\prime}-n\right) / 2} J_{n^{\prime}-n}\left(r \omega q^{-\frac{1}{2}} ; q\right) .
$$

This and other $q$-analogs of $m(2)$ matrix elements obey group-like addition theorems $[13,15,17]$.

The tensor product decomposition $\left(\omega_{1}\right) \otimes_{q}\left(\omega_{2}\right)$ of $(3.1)$ is not unique, [15]. The simplest decompositons are

$$
\left(\omega_{1}\right) \otimes_{q}\left(\omega_{2}\right) \equiv \sum_{p=-\infty}^{\infty} \oplus\left(\omega_{1} q^{p / 2}\right),
$$

and another decompostion obtained by replacing $\omega_{1}$ with $\omega_{2}$ on the right-hand side of (3.4). A special case of the identity relating the matrix elements of the operator $e_{q}\left(\beta F_{+}\right) E_{q}\left(\alpha F_{-}\right)$ with respect to the tensor product basis and with respect to the reduced basis is

$$
(-q)^{n} J_{x-n}\left(S q^{-y} ; q^{2}\right) J_{n}\left(R S q^{n} ; q^{2}\right)=\sum_{k=-\infty}^{\infty} q^{2 k} J_{k-n}\left(R q^{y} ; q^{2}\right) J_{x}\left(S q^{k-y} ; q^{2}\right) J_{k}\left(R q^{y+x} ; q^{2}\right),
$$

valid for $0<R q^{y+x+1}<1,0<R q^{y+1}<1,0 \leq S$ and $n, x, y$ integers. The case $S=q^{z}$ of this formula for $z$ an integer is the addition theorem for Hahn-Exton $q$-Bessel functions derived by Koelink using the theory of quantum groups, $[12,15]$.

(The computation of the matrix elements on the left hand side of this formula and in many formulas to follow is based heavily on the formal identities

$$
E_{q}(X+Y)=E_{q}(Y) E_{q}(X), \quad e_{q}(X+Y)=e_{q}(X) e_{q}(Y)
$$

valid for operators $X, Y$ such that $Y X=q X Y$. 
In [18] a $q$-analog of the oscillator algebra was introduced. This is the associative algebra generated by the four elements $H, E_{+}, E_{-}, \mathcal{E}$ that obey the commutation relations

$$
\left[H, E_{+}\right]=E_{+},\left[H, E_{-}\right]=-E_{-},\left[E_{+}, E_{-}\right]=-q^{-H} \mathcal{E},\left[\mathcal{E}, E_{ \pm}\right]=[\mathcal{E}, H]=0 .
$$

It admits a class of algebraically irreducible representations $\uparrow_{\lambda, \ell^{2}}$ where $\ell, \lambda$ are real numbers and $\ell>0$. A convenient orthogonal basis for the representation space is $\left\{f_{n}: n=\right.$ $0,1, \cdots\}$ where

$$
\begin{aligned}
E_{+} f_{n} & =\ell q^{-(n+1) / 2} f_{n+1}, \quad E_{-} f_{n}=\ell q^{-n / 2} \frac{1-q^{n}}{1-q} f_{n-1} \\
H f_{n} & =(\lambda+n) f_{n}, \quad \varepsilon f_{n}=\ell^{2} q^{\lambda-1} f_{n} .
\end{aligned}
$$

Here, $f_{n}=\sqrt{(q ; q)_{n} /(1-q)^{n}} e_{n}$. We have $E_{+}=\left(E_{-}\right)^{*}, H^{*}=H$ and $\mathcal{E}^{*}=\mathcal{E}$. The elements $\mathcal{C}=q q^{-H} \mathcal{E}+(q-1) E_{+} E_{-}$and $\mathcal{E}$ lie in the center of this algebra, and corresponding to the irreducible representation $\uparrow_{\lambda, \ell^{2}}$ we have $\mathcal{C}=\ell^{2} I, \mathcal{E}=\ell^{2} q^{\lambda-1} I$ where $I$ is the identity operator.

We define matrix elements of the "group" by

$$
E_{q}\left(\beta E_{+}\right) e_{q}\left(\alpha E_{-}\right) f_{n}=\sum_{n^{\prime}} T_{n^{\prime} n}^{(E+, e-)}(\alpha, \beta) f_{n^{\prime}}
$$

where

$$
\begin{aligned}
& T_{n^{\prime} n}^{(E+, e-)}(\alpha, \beta) \\
& =\frac{\left(q^{n^{\prime}+1} ; q\right)_{\infty}\left(q^{n-n^{\prime}+1} ; q\right)_{\infty}(\alpha \ell)^{n-n^{\prime}}}{(q ; q)_{\infty}\left(q^{n+1} ; q\right)_{\infty}(1-q)^{n-n^{\prime}}} q^{\left(n^{\prime}-n\right)\left(n^{\prime}+n+1\right) / 4}{ }_{1} \phi_{1}\left(\begin{array}{c}
q^{-n^{\prime}} \\
q^{n-n^{\prime}+1} ; q, \frac{\alpha \beta \ell^{2}}{1-q}
\end{array}\right) \\
& =\frac{\left(q^{n^{\prime}-n+1} ; q\right)_{\infty}(\beta \ell)^{n^{\prime}-n}}{(q ; q)_{\infty}} q^{\left(n^{\prime}-n\right)\left(n^{\prime}-3 n-3\right) / 4}{ }_{1} \phi_{1}\left(\begin{array}{c}
q^{-n} \\
q^{n^{\prime}-n+1}
\end{array} ;, \frac{\alpha \beta \ell^{2} q^{n^{\prime}-n}}{1-q}\right)
\end{aligned}
$$

Given the irreducible representations $\uparrow_{\lambda_{1}, \ell_{1}^{2}}$ and $\uparrow_{\lambda_{2}, \ell_{2}^{2}}$ we define the tensor product representation $\uparrow_{\lambda_{1}, \ell_{1}^{2}} \otimes \uparrow_{\lambda_{2}, \ell_{2}^{2}}$ by the operators $[18,19]$

$$
\begin{aligned}
F_{+} & =E_{+} \otimes q^{\frac{1}{2} H}+q^{-\frac{1}{2} H} \otimes E_{+}, F_{-}=E_{-} \otimes q^{\frac{1}{2} H}+q^{-\frac{1}{2} H} \otimes E_{-}\left(\kappa_{1} q^{H}+\kappa_{2}\right) \\
L & =H \otimes I+I \otimes H, \mathcal{F}=\mathcal{E} \otimes I+I \otimes \mathcal{E}=\left(\ell_{1}^{2} q^{\lambda_{1}-1}+\ell_{2}^{2} q^{\lambda_{2}-1}\right) I \otimes I
\end{aligned}
$$

where

$$
\kappa_{1}=-\frac{\ell_{1}^{2} q^{\lambda_{1}-1}}{\ell_{2}^{2}}, \quad \kappa_{2}=\frac{\ell_{1}^{2} q^{\lambda_{1}}+\ell_{2}^{2} q^{\lambda_{2}}}{\ell_{2}^{2} q^{\lambda_{2}}} .
$$

Then we have

$$
\left[L, F_{ \pm}\right]= \pm F_{ \pm},\left[F_{+}, F_{-}\right]=-\mathcal{F} q^{-L},\left[\mathcal{F}, F_{ \pm}\right]=[\mathcal{F}, L]=0
$$


This representation-dependent coproduct does not define the $q$-oscillator as a quantum algebra. The tensor product decomposition is

$$
\uparrow_{\lambda_{1}, \ell_{1}^{2}} \otimes \uparrow_{\lambda_{2}, \ell_{2}^{2}} \cong \sum_{s=0}^{\infty} \oplus \uparrow_{\lambda_{1}+\lambda_{2}+s, \tilde{\ell}_{s}^{2}}
$$

where $\tilde{\ell}_{s}^{2}=q^{-s}\left(\ell_{1}^{2} q^{-\lambda_{2}}+\ell_{2}^{2} q^{-\lambda_{1}}\right)$. Relating the matrix elements of the operator $E_{q}\left(\beta F_{+}\right) e_{q}\left(\alpha F_{-}\right)$ with respect to both the tensor product basis and the reduced basis we find $[18,19]$

$$
\frac{d^{n^{\prime}}(c q ; q)_{n^{\prime}}(d q ; q)_{\infty}}{(c d q ; q)_{\infty}(q ; q)_{m^{\prime}}(w ; q)_{n^{\prime}}}{ }_{1} \phi_{1}\left(\begin{array}{c}
q^{-m^{\prime}} \\
d q
\end{array} ; q, z d\right){ }_{2} \phi_{1}\left(\begin{array}{c}
q^{-n^{\prime}}, w^{-1} q^{1-n^{\prime}} \\
c q
\end{array} ; q, z q^{-1-m^{\prime}}\right)=
$$

$$
\begin{aligned}
& \sum_{s=0}^{m^{\prime}+n^{\prime}} \frac{(w ; q)_{s} w^{m^{\prime}-s}}{(q ; q)_{s}(q ; q)_{m^{\prime}+n^{\prime}-s}}{ }_{1} \phi_{1}\left(\begin{array}{c}
q^{s-m^{\prime}-n^{\prime}} \\
c d q
\end{array} ; q, \frac{z d q^{-s-n^{\prime}}}{w}\right) \times \\
& { }_{2} \phi_{1}\left(\begin{array}{c}
c^{-1} q^{-n^{\prime}}, w q^{s} \\
w
\end{array} ; q, c d q^{m^{\prime}+n^{\prime}-s+1}\right){ }_{2} \phi_{1}\left(\begin{array}{c}
q^{-n^{\prime}}, w q^{s} \\
w
\end{array} ; q, q^{m^{\prime}+n^{\prime}-s+1}\right) \text {. }
\end{aligned}
$$

Note that the representation $(\omega)$ of $m(2)$ can be considered as a representation of the $q$ oscillator algebra for which $\mathcal{E}=0$. We define the tensor product representation $(\omega) \otimes \uparrow_{\lambda, \ell^{2}}$ by

$$
\begin{aligned}
F_{+} & =E_{+} \otimes q^{\frac{1}{2} H}+q^{-\frac{1}{2} H} \otimes E_{+}, F_{-}=E_{-} \otimes q^{\frac{1}{2} H}+q^{-\frac{1}{2} H} \otimes E_{-} \\
L & =H \otimes I+I \otimes H, \mathcal{F}=I \otimes \mathcal{E} .
\end{aligned}
$$

By construction

$$
\left[L, F_{ \pm}\right]= \pm F_{ \pm},\left[F_{+}, F_{-}\right]=-\mathcal{F} q^{-L},\left[\mathcal{F}, F_{ \pm}\right]=[\mathcal{F}, L]=0
$$

The unique decomposition is [19]

$$
(\omega) \otimes \uparrow_{\lambda, \ell^{2}} \cong \oplus \sum_{\xi_{0} \in Z} \uparrow_{\lambda+\dot{\xi}_{0}, q^{-\xi_{0} \ell^{2}}} \oplus \sum_{s=0}^{\infty} R\left(\sqrt{1-q} q^{(\lambda+s) / 2} \omega, q^{-\lambda-s} \ell^{2} /(1-q) \omega^{2}, \lambda\right) .
$$

where $R$ is a family of irreducible representations of the $q$-oscillator algebra for which the spectrum of $H$ is unbounded both above and below. Thus the following identity, relating 
the matrix elements in the tensor product and reduced bases must hold:

$$
\begin{aligned}
& \frac{(q ; q)_{h}}{(q ; q)_{j-j^{\prime}}(q ; q)_{h-h^{\prime}}}{ }_{1} \phi_{1}\left(\begin{array}{c}
0 \\
q^{j-j^{\prime}+1} ; q,-y q^{h}
\end{array}\right){ }_{1} \phi_{1}\left(\begin{array}{c}
q^{-h^{\prime}} \\
q^{h-h^{\prime}+1} ; q, z q^{-j^{\prime}}
\end{array}\right) \\
& =\sum_{\xi=-\infty}^{\min \left(j+h, j^{\prime}+h^{\prime}\right)} \frac{(q ; q)_{j+h-\xi}(y / z)^{j^{\prime}-\xi} q^{\left[\left(j+h-j^{\prime}-h^{\prime}\right)\left(j+h-j^{\prime}-h^{\prime}-1\right)-\xi(\xi+1)-\xi(j+h)\right] / 2}}{(q ; q)_{j+h-j^{\prime}-h^{\prime}}(q ; q)_{j^{\prime}-\xi}(q ; q)_{j-\xi}\left(-y q^{\xi} / z ; q\right)_{\infty}} \\
& \times_{1} \phi_{1}\left(\begin{array}{c}
q^{-h} \\
q^{j-\xi+1}
\end{array} ; q,-y q^{j+h+1} / z\right){ }_{1} \phi_{1}\left(\begin{array}{c}
q^{-h^{\prime}} \\
q^{j^{\prime}-\xi+1}
\end{array} ; q,-y q^{j^{\prime}+h^{\prime}+1} / z\right) \\
& \times q^{h\left(j^{\prime}-j\right)+j^{\prime}\left(j^{\prime}+1\right) / 2}{ }_{1} \phi_{1}\left(\begin{array}{c}
q^{\xi-j^{\prime}-h^{\prime}} \\
q^{j+h-j^{\prime}-h^{\prime}+1} ; q, z q^{-\xi+j+h-j^{\prime}-h^{\prime}}
\end{array}\right) \\
& +\sum_{s=\mathbf{0}}^{\infty} \frac{(q ; q)_{s}(z / y)^{s-h}(1-q)^{j+h-j^{\prime}-h^{\prime}} q^{-s\left(j^{\prime}+h^{\prime}\right)+h\left(h+j^{\prime}\right)+h^{\prime}\left(h^{\prime}-1\right) / 2}}{(q ; q)_{j+h-j^{\prime}-h^{\prime}}(q ; q)_{s-h}(q ; q)_{s-h^{\prime}}\left(-z q^{-j^{\prime}-h^{\prime}-s} / y ; q\right)_{\infty}} \\
& \times_{1} \phi_{1}\left(\begin{array}{c}
q^{-h} \\
q^{s-h+1}
\end{array} ; q,-z q^{-j-h+1} / y\right){ }_{1} \phi_{1}\left(\begin{array}{c}
q^{-h^{\prime}} \\
q^{s-h^{\prime}+1}
\end{array} ; q,-z q^{-j^{\prime}-h^{\prime}+1} / y\right) \\
& \times(-1)^{h+h^{\prime}}{ }_{1} \phi_{1}\left(\begin{array}{c}
-z q^{-s-j^{\prime}-h^{\prime}} / y \\
q^{j+h-j^{\prime}-h^{\prime}+1}
\end{array} ; q,-y q^{s}\right)
\end{aligned}
$$

where $y / z>0, h, h^{\prime}, \pm j, \pm j^{\prime}=0,1, \cdots$.

4. Discrete models of $m(2)$ and oscillator algebra representations. In this section we study representations of a number of discrete $q$-analogs of $m(2)$ and the oscillator algebra. We start with discrete $m_{\epsilon}(2)$ where $\epsilon^{N}=1$ and $\epsilon$ is a primitive $N$ th root of unity. The generators are $\epsilon^{ \pm H}, E_{ \pm}$with relations

$$
\epsilon \epsilon^{H} E_{-}=E_{-} \epsilon^{H}, \quad \epsilon^{H} E_{+}=\epsilon E_{+} \epsilon^{H}, \quad\left[E_{+}, E_{-}\right]=0
$$

and $\epsilon^{H} \epsilon^{-H}=I$. We consider $N$-dimensional irreducible representations $(\omega, \bar{\omega})_{N}$ of $m_{\epsilon}(2)$, characterized by the nonzero complex numbers $\omega, \bar{\omega}$. The complex representation space has basis vectors $f_{\ell}, \ell=0,1, \cdots, N-1, \bmod N$, such that

$$
E_{+} f_{\ell}=\omega f_{\ell+1}, E_{-} f_{\ell}=\bar{\omega} f_{\ell-1}, \epsilon^{H} f_{\ell}=\epsilon^{\ell} f_{\ell}
$$

and $C f_{\ell}=\omega \bar{\omega} I$ where $C \equiv E_{+} E_{-}$is an invariant operator. We define the tensor product $\left(\omega_{1}, \bar{\omega}_{1}\right)_{N} \otimes\left(\omega_{2}, \bar{\omega}_{2}\right)_{N}$ by

$$
F_{ \pm}=E_{ \pm} \otimes I+I \otimes E_{ \pm}, \quad \epsilon^{L}=\epsilon^{H} \otimes \epsilon^{H} .
$$

Determining the spectrum of the invariant operator $F_{+} F_{-}$, we find

$$
\left(\omega_{1}, \bar{\omega}_{1}\right)_{N} \otimes\left(\omega_{2}, \bar{\omega}_{2}\right)_{N} \cong \sum_{s=0}^{N-1} \oplus\left(\omega_{1}+\omega_{2} \epsilon^{-s}, \bar{\omega}_{1}+\bar{\omega}_{2} \epsilon^{s}\right)_{N}
$$


Similarly, we define the "twisted" tensor product $\left(\omega_{1}, \bar{\omega}_{1}\right)_{N} \otimes_{\epsilon}\left(\omega_{2}, \bar{\omega}_{2}\right)_{N}$ by

$$
F_{+}=E_{+} \otimes \epsilon^{H}+I \otimes E_{+}, F_{-}=E_{-} \otimes I+\epsilon^{-H} \otimes E_{-}, \epsilon^{L}=\epsilon^{H} \otimes \epsilon^{H} .
$$

Now the tensor product expansion is

$$
\left(\omega_{1}, \bar{\omega}_{1}\right)_{N} \otimes_{\epsilon}\left(\omega_{2}, \bar{\omega}_{2}\right)_{N} \cong \sum_{s=0}^{N-1} \oplus\left(\left(\omega_{1}^{N}+\omega_{2}^{N}\right)^{N^{-1}} \epsilon^{s},\left(\bar{\omega}_{1}^{N}+\bar{\omega}_{2}^{N}\right)^{N^{-1}}\right)_{N}
$$

For these cases and the examples to follow, operators such as $E_{q}\left(\beta F_{+}\right) e_{q}\left(\alpha F_{-}\right)$no longer make sense. Instead, one can obtain identities relating the matrix elements of operators $F_{+}^{n} F_{-}^{m}$ with respect to the tensor product basis and the reduced basis.

For the same $\epsilon$ with $\epsilon^{N}=1$ we can define the discrete oscillator algebra with generators $E_{ \pm}, \epsilon^{ \pm H}, \mathcal{E}$ and relations

$$
\left[E_{+}, E_{-}\right]=-\epsilon^{-H} \mathcal{E}, \epsilon \epsilon^{H} E_{-}=E_{-} \epsilon^{H}, \epsilon^{H} E_{+}=\epsilon E_{+} \epsilon^{H}
$$

with $\mathcal{E}$ in the center. Here, $C=\epsilon \epsilon^{-H} \mathcal{E}+(\epsilon-1) E_{+} E_{-}$commutes with all elements of the algebra. A family of finite dimensional irreducible representations is given by $\uparrow_{\lambda, \ell^{2}}$ where $\ell>0$ and $\lambda$ is an integer, $\bmod N$. A convenient orthogonal basis for the representation space is $\left\{f_{n}: n=0,1, \cdots, N-1, \bmod N\right\}$ where

$$
\begin{aligned}
E_{+} f_{n} & =\ell \epsilon^{-n-1} f_{n+1}, E_{-} f_{n}=\ell \frac{1-\epsilon^{n}}{1-\epsilon} f_{n-1} \\
\epsilon^{-H} f_{n} & =\epsilon^{-\lambda-n} f_{n}, \varepsilon f_{n}=\ell^{2} \epsilon^{\lambda-1} f_{n}, C f_{n}=-\ell^{2} f_{n} .
\end{aligned}
$$

A second family of representations $R(\ell, \alpha, \delta, \lambda)$ is defined by

$$
\begin{gathered}
E_{+} f_{n}=\alpha \ell \epsilon^{-n-1} f_{n+1}, E_{-} f_{n}=\frac{\ell}{\alpha} \frac{1+\delta \epsilon^{-n}}{1-\epsilon} f_{n-1} \\
\epsilon^{-H} f_{n}=\epsilon^{-\lambda-n} f_{n}, \varepsilon f_{n}=\ell^{2} \epsilon^{\lambda-1} \delta f_{n}, C f_{n}=-\ell^{2} f_{n} .
\end{gathered}
$$

Restricting to the case $N$ odd for convenience, we can define the tensor product representation $(\omega, \bar{\omega})_{N} \otimes \uparrow_{\lambda, \ell^{2}}$ by the relations

$$
F_{ \pm}=E_{ \pm} \otimes \epsilon^{H / 2}+\epsilon^{-H / 2} \otimes E_{ \pm}, \epsilon^{L}=\epsilon^{H} \otimes \epsilon^{H}, \mathcal{F}=I \otimes \mathcal{E} .
$$

Computing the spectrum of $C$ we find

$$
(\omega, \bar{\omega})_{N} \otimes \uparrow_{\lambda, \ell^{2}} \cong \sum_{s=0}^{N-1} \oplus R\left(-c_{s}, \frac{\left(1+\frac{\ell^{N}}{\omega^{N}}\right)^{N^{-1}} \omega}{\sqrt{1-c_{s}}}, \frac{-\ell^{2}}{c_{s}}, \lambda\right)
$$

where $c_{s}=c_{0} \epsilon^{s}$ and $c_{0}^{N}=\ell^{2 N}+(\epsilon-1)^{N}\left(\omega^{2 N}+\omega^{N} \ell^{N}\right)$. 
For $0<q<1$ the generators of the discrete pseudo-euclidean algebra are $q^{ \pm H}, E_{+}, E_{-}$ with relations

$$
q^{H} E_{+}=q E_{+} q^{H}, \quad q q^{H} E_{-}=E_{-} q^{H}, \quad\left[E_{+}, E_{-}\right]=0 .
$$

We consider the following class of irreducible representations $(\omega, \bar{\omega})$ for this algebra, characterized by the nonzero complex numbers $\omega, \bar{\omega}$. The Hilbert space consists of complex functions $f(x)$ with domain $x=q^{n}, n=0, \pm 1, \pm 2, \cdots$ and such that $(f, f)<\infty$, where the inner product is

$$
(f, g)=\sum_{n=-\infty}^{\infty} f\left(q^{n}\right) \bar{g}\left(q^{n}\right)
$$

The action of the algebra on this Hilbert space is given by the operators

$$
E_{+}=\omega x, \quad E_{-}=\frac{\bar{\omega}}{x}, \quad q^{H} f(x)=f(q x)
$$

and $C f_{\ell}=\omega \bar{\omega} I$ where $C \equiv E_{+} E_{-}$is an invariant operator. We define the tensor product $\left(\omega_{1}, \bar{\omega}_{1}\right)_{q} \otimes\left(\omega_{2}, \bar{\omega}_{2}\right)_{q}$ by

$$
F_{ \pm}=E_{ \pm} \otimes I+I \otimes E_{ \pm}, \quad q^{L}=q^{H} \otimes q^{H} .
$$

Determining the spectrum of the invariant operator $F_{+} F_{-}$we find

$$
\left(\omega_{1}, \bar{\omega}_{1}\right)_{q} \otimes\left(\omega_{2}, \bar{\omega}_{2}\right)_{q} \cong \sum_{m=-\infty}^{\infty} \oplus\left(\omega_{1}+\omega_{2} q^{-m}, \bar{\omega}_{1}+\bar{\omega}_{2} q^{m}\right)_{q}
$$

Similarly, we define the "twisted" tensor product $\left(\omega_{1}, \bar{\omega}_{1}\right)_{q} \otimes_{q}\left(\omega_{2}, \bar{\omega}_{2}\right)_{q}$ by

$$
F_{+}=E_{+} \otimes q^{H}+I \otimes E_{+}, F_{-}=E_{-} \otimes I+q^{-H} \otimes E_{-}, q^{L}=q^{H} \otimes q^{H} .
$$

Now the tensor product expansion is not unique; one of the the simplest expansion is

$$
\left(\omega_{1}, \bar{\omega}_{1}\right)_{q} \otimes_{q}\left(\omega_{2}, \bar{\omega}_{2}\right)_{q} \cong \frac{1}{2 \pi} \int_{0}^{2 \pi} \oplus\left(\omega_{1}, \frac{e^{i \theta}}{\omega_{1}}\right) d \theta
$$

For this algebra the matrix elements can be expressed as kernels of integral operators [20].

The discrete pseudo-oscillator algebra has generators $q^{ \pm H}, E_{+}, E_{-}, \mathcal{E}$ which obey the relations

$$
\begin{gathered}
E_{+} q^{-H}=q q^{-H} E_{+}, \quad q E_{-} q^{-H}=q^{-H} E_{-}, \quad\left[E_{+}, E_{-}\right]=-q^{-H} \mathcal{E} \\
{\left[\mathcal{E}, E_{ \pm}\right]=\left[\mathcal{E}, q^{-H}\right]=0 .}
\end{gathered}
$$


We consider the irreducible representations $\uparrow_{q^{\lambda}, \ell^{2}}$ for this algebra, characterized by the positive number $\ell$ and real number $\lambda$. The Hilbert space consists of complex functions $f(x)$ with domain $x=q^{n}, n=0, \pm 1, \pm 2, \cdots$ and such that $(f, f)<\infty$, where the inner product is

$$
(f, g)=\sum_{n=-\infty}^{\infty} f\left(q^{n}\right) \bar{g}\left(q^{n}\right) .
$$

The action of the algebra on this Hilbert space is given by the operators

$$
\begin{aligned}
E_{+} & =\ell x, \quad E_{-}=-\frac{\ell}{(1-q) x}\left(1-T_{x}^{-1}\right), \quad q^{-H} f(x)=f\left(q^{-1} x\right) q^{-\lambda} \\
\mathcal{E} & =q^{\lambda-1} \ell^{2}, \quad C=q q^{-H} \mathcal{E}+(q-1) E_{+} E_{-}=\ell^{2}
\end{aligned}
$$

Note that the representation $(\omega, \bar{\omega})_{q}$ of the discrete pseudo-euclidean algebra can be considered as a representation of the discrete pseudo-oscillator algebra for which $\mathcal{E}=0$. We define the discrete tensor product $(\omega, \bar{\omega})_{q} \otimes \uparrow_{q^{\lambda}, \ell^{2}}$ by

$$
F_{+}=E_{+} \otimes q^{H}+I \otimes E_{+}, F_{-}=E_{-} \otimes I+q^{-H} \otimes E_{-}, q^{L}=q^{H} \otimes q^{H}, \mathcal{F}=I \otimes \mathcal{E} .
$$

The decomposition is not unique; one of the simplest expansions is

$$
(\omega, \bar{\omega})_{q} \otimes \uparrow_{q^{\lambda}, \ell^{2}} \cong \sum_{n=-\infty}^{\infty} \oplus \uparrow_{\frac{q^{\lambda-n+\frac{1}{2} \ell}}{\omega}, \omega \ell q^{n-\frac{1}{2}}} .
$$

As a final example we consider another version of the discrete pseudo-euclidean algebra, the algebra with generators $\epsilon^{ \pm H}, E_{ \pm}$and relations (4.1), where now $|\epsilon|=1$ but $\epsilon^{N} \neq 1$ for any integer $N$. A model of the irreducible representation $(\omega)_{\epsilon}$ for $\omega$ a nonzero complex number is given by the operators

$$
E_{+}=z, E_{-}=\frac{\omega}{z}, \epsilon^{H} f(z)=f(\epsilon z), z=\epsilon^{n},
$$

where $n=0, \pm 1, \pm 2, \cdots$, and the inner product is

$$
(f, g)=\sum_{n=-\infty}^{\infty} f\left(\epsilon^{N}\right) \overline{g\left(\epsilon^{N}\right)} .
$$

The tensor product $\left(\omega_{1}\right)_{\epsilon} \otimes_{\epsilon}\left(\omega_{2}\right)_{\epsilon}$ is defined by

$$
F_{ \pm}=E_{ \pm} \otimes \epsilon^{-H}+\epsilon^{H} \otimes E_{ \pm}, \epsilon^{L}=\epsilon^{H} \otimes \epsilon^{H} .
$$

The unique decomposition is

$$
\left(\omega_{1}\right)_{\epsilon} \otimes_{\epsilon}\left(\omega_{2}\right)_{\epsilon} \cong \frac{1}{2 \pi} \int_{0}^{2 \pi} \oplus\left(e^{i \theta}\right)_{\epsilon} d \theta .
$$




\section{REFERENCES}

1. G. Darboux (1889), Lecons sur la Théorie Génerale des Surfaces et les Applications Géométrique du Calcul Infinitésimal, vol. 2, Entry 408, Gautier Villars et Fils, Paris.

2. L. Infeld and T.E. Hull (1951), The Factorization Method, Rev. Mod. Phys. 23, 21-68.

3. W. Miller (1964), On Lie algebras and some special functions of mathematical physics, American Mathematical Society Memoir no. 50, Providence, Rhode Island.

4. W. Miller (1968), Lie Theory and Special Functions, Academic Press, New York.

5. W. Miller (1969), Lie theory and difference equations, J. Math. Anal. Appl. 28, 383-399.

6. W. Miller (1970), Lie theory and q-difference equations, SIAM J. Math. Anal. 1, 171-188.

7. V. Spiridonov and A. Zhedanov (1994), Discrete Darboux transformations, Discrete time Toda lattice, and the Askey-Wilson polynomials, preprint CRM-1929, Université de Montréal.

8. V.B. Matveev (1979), Darboux transformation and explicit solutions of differential-difference and differential-difference evolution equations. I, Lett. Math. Phys. 3, 217-222.

9. G. Gasper and M. Rahman (1990), Basic Hypergeometric Series, Cambridge University Press, Cambridge.

10. N. Ja. Vilenkin (1968), Special Functions and the Theory of Group Representations, American Mathematical Society, Providence, Rhode Island.

11. W. Miller (1965), The special function theory of occupation number space, Comm. Pure Appl. Math. 18, 679-696.

12. H.T. Koelink (1991), On quantum groups and q-special functions, thesis University of Leiden.

13. T.H. Koornwinder and R.F. Swarttouw (1991), On q-analogues of the Fourier and Hankel transforms group, (to appear in Trans. American Math. Soc.).

14. L.L. Vaksman and L.I. Korogodskï (1989), An algebra of bounded functions on the quantum group of the motions of the plane, and q-analogues of Bessel functions, Soviet Math. Dokl. 39, 173-177.

15. E.G. Kalnins, S. Mukherjee and W. Miller (1994), Models of q-algebra representations: The group of plane motions, SIAM J. Math. Anal. 25, 513-527.

16. R. Floreanini and L. Vinet (1992), Addition formulas for q-Bessel functions, J. Math. Phys. 33, 2984-2988.

17. H.T. Koelink (1993), A basic analogue of Graf's addition formula and related formulas, preprint Katholieke Universiteit Leuven.

18. E.G. Kalnins, H.L. Manocha and W. Miller (1992), Models of q-algebra representations: Tensor products of special unitary and oscillator algebras, J. Math. Phys. 33, 2365-2383.

19. E.G. Kalnins, S. Mukherjee and W. Miller (1993), Models of q-algebra representations: Matrix elements of the q-oscillator algebra, J. Math. Phys. 34, 5333-5356.

20. E.G. Kalnins and W. Miller (1994), Models of q-algebra representations: q-integral transforms and "addition theorems", J. Math. Phys. (to appear). 